\title{
A urinary biosignature for mitochondrial myopathy, encephalopathy, lactic acidosis and stroke like episodes (MELAS)
}

\section{Esterhuizen, Karien}

2019-03

Esterhuizen , K , Lindeque , J Z, Mason , S , van der Westhuizen , F H, Suomalainen , A , Hakonen, A H , Carroll , C J , Rodenburg , R J , de Laat, P B , Janssen , M C H , Smeitink, J A M \& Louw , R 2019 , ' A urinary biosignature for mitochondrial myopathy, encephalopathy, lactic acidosis and stroke like episodes (MELAS) ' , Mitochondrion , vol. 45 , pp. 38-45 . https://doi.org/10.1016/j.mito.2018.02.003

http://hdl.handle.net/10138/300712

https://doi.org/10.1016/j.mito.2018.02.003

cc_by_nc_nd

acceptedVersion

Downloaded from Helda, University of Helsinki institutional repository.

This is an electronic reprint of the original article.

This reprint may differ from the original in pagination and typographic detail.

Please cite the original version. 


\section{Accepted Manuscript}

A urinary biosignature for mitochondrial myopathy, encephalopathy, lactic acidosis and stroke like episodes (MELAS)

Karien Esterhuizen, J. Zander Lindeque, Shayne Mason, Francois H. van der Westhuizen, Anu Suomalainen, Anna H. Hakonen, Christopher J. Carroll, Richard J. Rodenburg, Paul B. de Laat,

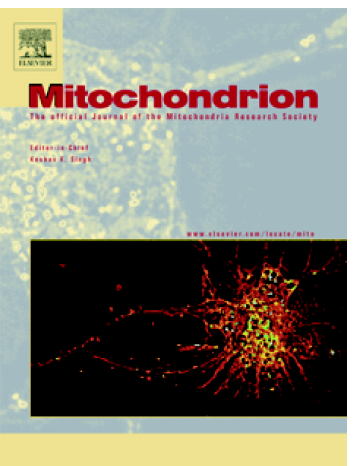
Mirian C.H. Janssen, Jan A.M. Smeitink, Roan Louw

PII: S1567-7249(17)30237-4

DOI: doi:10.1016/j.mito.2018.02.003

Reference: MITOCH 1269

To appear in: Mitochondrion

Received date: 17 August 2017

Revised date: 27 January 2018

Accepted date: 15 February 2018

Please cite this article as: Karien Esterhuizen, J. Zander Lindeque, Shayne Mason, Francois H. van der Westhuizen, Anu Suomalainen, Anna H. Hakonen, Christopher J. Carroll, Richard J. Rodenburg, Paul B. de Laat, Mirian C.H. Janssen, Jan A.M. Smeitink, Roan Louw , A urinary biosignature for mitochondrial myopathy, encephalopathy, lactic acidosis and stroke like episodes (MELAS). The address for the corresponding author was captured as affiliation for all authors. Please check if appropriate. Mitoch(2018), doi:10.1016/j.mito.2018.02.003

This is a PDF file of an unedited manuscript that has been accepted for publication. As a service to our customers we are providing this early version of the manuscript. The manuscript will undergo copyediting, typesetting, and review of the resulting proof before it is published in its final form. Please note that during the production process errors may be discovered which could affect the content, and all legal disclaimers that apply to the journal pertain. 


\section{A urinary biosignature for mitochondrial myopathy,}

\section{encephalopathy, lactic acidosis and stroke like episodes (MELAS)}

Karien Esterhuizen ${ }^{1}$, J. Zander Lindeque ${ }^{1}$, Shayne Mason ${ }^{1}$, Francois H. van der Westhuizen ${ }^{1}$, Anu Suomalainen ${ }^{2}$, Anna H. Hakonen ${ }^{2}$, Christopher J. Carroll ${ }^{2}$, Richard J. Rodenburg ${ }^{3}$, Paul B. de Laat ${ }^{3}$, Mirian C.H. Janssen ${ }^{3}$, Jan A.M. Smeitink ${ }^{3}$ and Roan Louw ${ }^{1}$

${ }^{1}$ Mitochondria Research Laboratory, Centre for Human Metabolomics, North-West University, Potchefstroom, South Africa

${ }^{2}$ Research Programs Unit, Molecular Neurology, Biomedicum-Helsinki, University of Helsinki, Helsinki, Finland.

${ }^{3}$ Radboud Center for Mitochondrial Medicine, Department of Pediatrics, Radboud University Nijmegen Medical Center, Nijmegen, The Netherlands.

Corresponding Author: Roan Louw

Email: Roan.Louw@nwu.ac.za

Postal address:

North-West University

Potchefstroom Campus

Private Bag X6001

Potchefstroom

2520

South Africa 


\section{Abstract}

We used a comprehensive metabolomics approach to study the altered urinary metabolome of two mitochondrial myopathy, encephalopathy lactic acidosis and stroke like episodes (MELAS) cohorts carrying the $\mathrm{m} .3243 \mathrm{~A}>\mathrm{G}$ mutation.

The first cohort were used in an exploratory phase, identifying 36 metabolites that were significantly perturbed by the disease. During the second phase, the 36 selected metabolites were able to separate a validation cohort of MELAS patients completely from their respective control group, suggesting usefulness of these 36 markers as a diagnostic set. Many of the 36 perturbed metabolites could be linked to an altered redox state, fatty acid catabolism and one-carbon metabolism. However, our evidence indicates that, of all the metabolic perturbations caused by MELAS, stalled fatty acid oxidation prevailed as being particularly disturbed.

The strength of our study was the utilization of five different analytical platforms to generate the robust metabolomics data reported here. We show that urine may be a useful source for disease-specific metabolomics data, linking, amongst others, altered one-carbon metabolism to MELAS. The results reported here are important in our understanding of MELAS and might lead to better treatment options for the disease.

\section{Keywords}

MELAS; m.3243A>G; metabolomics; mutation; metabolism; mtDNA 


\section{INTRODUCTION}

Mitochondrial diseases are one of the most common inborn errors of metabolism, with a minimum prevalence of 1:5000 (Gorman et al., 2015; Schaefer et al., 2004). Mitochondrial myopathy, encephalopathy, lactic acidosis and stroke like episodes, better known as MELAS (MIM 540000), is one such a mitochondrial disease and was first described in 1984 as a "neurodegenerative disease caused by the decreased ability of cells to produce sufficient energy in the form of ATP" (Pavlakis et al., 1984). In 1990, the MELAS phenotype was associated with a specific mutation, now known as the m.3243A>G mutation (Goto et al., 1990). This mutation is a point mutation on position 3243 of the mitochondrial DNA (mtDNA), where an adenine $(A)$ is substitute $d$ with a guanine $(G)$ in the MT-TL 1 gene. The latter gene encodes for a transfer ribonucleic acid (tRNA), which recognizes the codons UUA and UUG for the amino acid leucine in mitochondrial translation (Scaglia and Northrop, 2006; Kami et al., 2012). The mutation thus disturbs mitochondrial protein synthesis, ultimately affecting Complexes I, III, IV and V of the oxidative phosphorylation (OXPHOS) system. Although almost $80 \%$ of all MELAS cases are caused by the m.3243A>G mutation, other mtDNA mutations have later been associated with the MELAS phenotype, including the m.3244G $>A, m .3258 T>C, m .3271 T>C, m .3291 T>C$ and m.8356T $>C$ mutation (Ruiz-Pesini, 2007; Scaglia and Northrop, 2006).

Patients diagnosed with MELAS can also present with a wide range of symptoms, varying in degree of prevalence and severity. However, in this study, a MELAS phenotype was defined as a patient carrying the m.3243A>G mutation AND that had at least one stroke like episode. Although late onset MELAS does occur, these patients were not included in this study. Initially, the m.3243A>G mutation was specifically associated with MELAS, however, over time various other phenotypes have also been linked to this mutation, including maternally inherited diabetes and deafness (MIDD), progressive external ophthalmoplegia (PEO) and mitochondrial recessive ataxia syndrome (MIRAS) (Scaglia and Northrop, 2006; Manwaring et al., 2007). 
A recent study attempted to identify mechanistic differences between different m.3243A>G phenotypes using nuclear magnetic resonance (NMR) spectroscopy. With this single metabolomics technique, the authors were able to identify six urinary metabolites (all decreased in the patients, compared to the controls) that were able to distinguish MELAS patients from controls (Hall et al., 2015). The markers included N-methyl nicotinamide, hippurate, creatinine, 4-cresyl sulfate, glycolate, and a marker with unconfirmed identity, possibly 4-hydroxyphenylacetic acid. Although these markers point towards altered phenylalanine and tyrosine metabolism, arginine metabolism (creatinine) and a connection to gut microbial metabolism (4-cresyl sulfate and glycolate), the exact mechanism(s) for these changes remains elusive. Here we aimed to extend these findings on MELAS by investigating the urine metabolome of MELAS patients using a multi-platform metabolomics approach. With a larger metabolome coverage, additional metabolites affected in the MELAS patients, compared to healthy controls, can potentially be identified. This can potentially provide a more extensive and improved biochemical understanding of the metabolic perturbations resulting from this specific phenotype.

\section{METHODS}

\subsection{Patients and Ethics}

Urine samples of two cohorts of genetically confirmed m.3243A>G MELAS patients and healthy controls were obtained from the Radboud University Medical Center in Nijmegen, The Netherlands and the University of Helsinki, Finland. To ensure group homogeneity, exclusion criteria were applied to the original cohorts, namely, 1) controls that received either medication or supplementation at the time of sample collection; 2 ) controls with a medical condition (such as diabetes, hypertension, etc.) Characteristics of the patients and controls are summarized in Table s 1 and 2, including data on the urinary epithelial cell $\mathrm{m} .3243 \mathrm{~A}>\mathrm{G}$ mutation load measured in the patients. 
The study complied with all applicable institutional guidelines and terms of the Declaration of Helsinki of 1975 (as revised in 2013) for investigation of human participants. Ethical approval was obtained from the Health Research Ethics Committee (HREC) of the North-West University (NWU00170-13-S1). All the patients as well as the controls provided written informed consent for their urine samples to be used for research purposes.

\subsection{Metabolic Profiling}

A multi-platform metabolomics approach, which consisted of targeted as well as untargeted techniques, was used to analyze the urine samples of the MELAS patients and healthy controls in order to obtain an extensive metabolomics data set. Targeted analyses consisted of liquid chromatography tandem-mass spectrometry (LC-MS/MS) while untargeted analyses consisted of gas chromatography-mass spectrometry (GC-MS), nuclear magnetic resonance (NMR) spectroscopy and liquid chromatography mass spectrometry with ion mobility (LC-IM-MS) - in positive and negative ionization mode. The protocols for the metabolomics techniques and sample preparation used are discussed in detail in the SI.

\subsection{Statistical analyses}

Data matrixes obtained from the GC-MS, LC-MS/MS, NMR and the two LC-IM-MS platforms were pre-processed individually and were then combined into one single metabolomics data set (pre-processing details are given in the $\mathrm{SI}$ ). The data were normalized to creatinine, as discussed in the SI. The data was then log-transformed before univariate and multivariate statistical analyses. Univariate analyses (student t-test and effect size) were performed in Excel 2013 (Microsoft Inc.), while the multivariate analyses [principle component analysis (PCA) and heatmaps] were done using Matlab (Mathworks, Natick, Ma) and MetaboAnalyst 3.0 (www.metaboanalyst.ca), respectively. A feature with a t-test $p$ value of $<0.05$ and an effect size $d$ value of $>0.8$ was considered important, 
thus showing statistical significant and practical differences between the patient group and the healthy controls. The covariance and discriminatory power of the important metabolites were visualized with PCA, heatmaps and a volcano plot. The identity of the important features were determined from matching the spectral information (NMR), retention time, high resolution mass and/or fragmentation spectra (MS) to in-house and commercial libraries as well as public databases. Confidence levels for each identity were included (Fiehn et al., 2007) as well as the analytical platform used to detect the metabolite (Table 3).

\section{RESULTS}

\subsection{Exploratory phase}

The Nijmegen cohort were utilized for the exploratory phase, i.e. searching for a biosignature for MELAS. Univariate measures - student's t-test $(p<0.05)$ and effect size $(d>0.8)-$ highlighted 36 metabolites that differed significantly between the MELAS patients and healthy controls (Table 3). All but four of the 36 urinary metabolites were increased in the MELAS patients (Fig. 1B +C), with only sarcosine, glycerol, hydroxylamine and 6-azathymine being decreased. The visualized covariance of these metabolites (Fig. 1A) resulted in separate grouping of the MELAS patients and controls (albeit with some overlap). The PCA score s plot show that the MELAS patients are more homogenous than the controls - indicating a more defined metabolic profile in comparison to the controls. A Venn diagram (Fig. 2) illustrates the contribution of each analytical platform used in this study towards the final 36 important metabolites detected. Although a large number of features were detected using each analytical platform, only those metabolites that differed significantly between the MELAS and control groups and could be identified/partially identified were considered - forming the final metabolic fingerprint.

\subsection{Validation phase}


The Helsinki cohort were used for the validation phase of this study. After identifying the 36 metabolites for MELAS using the Nijmegen cohort during the exploratory phase, data on the same 36 metabolites were extracted from the Helsinki cohort and used for the validation phase. The visualized covariance of these metabolites (Fig. 3) showed that the 36 metabolite fingerprint strongly separated MELAS patients from controls in a different sample set.

\section{DISCUSSION}

We report here a multi-platform metabolomics investigation of the urinary metabolome of MELAS patients carrying the $\mathrm{m} .3243 \mathrm{~A}>\mathrm{G}$ mutation. We show that urine may be a useful source for disease-specific metabolomics data. Furthermore, we identified a 36 metabolite fingerprint that partially-separated the patients from the controls in two separate cohorts, suggesting usefulness of these 36 separate markers as a diagnostic set.

Several of the affected pathways identified by our metabolomics approach was disturbed by redox-status (Fig. 4), with increased levels of lactic acid, pyruvic acid, acetoacetic acid and the branched chain amino acids (leucine, , isoleucine). These metabolites have previously been linked to mitochondrial diseases in various studies, using different mitochondrial disease models ( Esterhuizen et al., 2017) and fits with an altered $\mathrm{NAD}^{+} / \mathrm{NADH}$ ratio, now considered key in the development of mitochondrial disease (Khan et al., 2014). Cofactors NADH and FADH ${ }_{2}$ are formed in the tricarboxylic acid (TCA) cycle and serve as electron carriers onto the complexes of the OXPHOS system. When a defect in any one of the components of the OXPHOS system occurs, the flow of electrons along this chain is hindered. This perturbed flow results in altered levels of oxidized cofactors (NAD', FADH) to reduced cofactors ( $\mathrm{NADH}, \mathrm{FADH}$ ), i.e. a redox imbalance (Brière et al., 2004; Khan et al., 2014; Naviaux, 2014; Reinecke et al., 2009; Smeitink et al., 2006), affecting dehydrogenase enzymes. A redox imbalance could explain the altered lactic and pyruvic acid levels detected as lactate dehydrogenase, a redox dependent enzyme, is responsible for the interconversion of these two 
metabolites. The lactate/pyruvate ratio, an indicator of cytosolic redox state (Munnich et al., 1996) was slightly increased in the urine of MELAS patients, compared to the controls, although not statistically significant $(P=0.2)$. Furthermore, since branched chain amino acids are ultimately metabolized to their respective $\alpha$-keto acids through the branched-chain $\alpha$-keto acid dehydrogenase complex (BCKDC), altered BCKDC activity, caused by a redox imbalance, could elucidate the accumulation of the isoleucine and leucine detected in the MELAS cohort. Another point of interest was the ketone body acetoacetic acid, which is converted to 3-hydroxybutyric acid by 3hydroxybutyric acid dehydrogenase. Since the activity of this enzyme is also dependent on the redox balance, an altered redox balance caused by MELAS could explain the increased levels of acetoacetic acid found in our MELAS patient group. Thus our findings suggest that urine reflects the ove rall redox-balance of the body.

Currently receiving a lot of attention in the field of mitochondrial disease research is an intricate web of pathways consisting of one-carbon metabolism, the methylation cycle and the transsulfuration pathway. It has been hypothesized that these pathways could be affected in defective OXPHOS systems, but it was not until recent studies that this intricate web of pathways was positively associated with the disease (Bao et al., 2016; Nikkanen et al., 2016). In addition to a redox imbalance, our data is also supportive of alterations in this cluster of pathways, with increased levels of, cysteine, trimethylglycine (betaine), taurine, and trimethylamine $\mathrm{N}$-oxide (TMAO) detected (Fig. 4D and Table 3). Firstly, cysteine is metabolized to taurine through a cofactor-dependent reaction, thus influencing the taurine levels detected in the MELAS patients. Secondly, during the methylation cycle, homocysteine is converted to methionine through a reaction where choline is converted to trimethylglycine (betaine), ultimately producing dimethylglycine. Finally, choline additionally produces trimethylamine (TMA), which is further broken down to TMAO and dimethylamine. Thus the results reported here not only indicate affected one-carbon metabolism, but also an altered 
trans-sulfuration pathway and methylation cycle, explaining the altered levels of all these metabolites observed in this study.

Another component of the metabolism that seems to be strongly affected by MELAS is fatty acid oxidation. We hypothesize that stalled beta-oxidation is responsible for the altered levels of glycerol, butyric/valeric acid (short chain fatty acid), caproic/caprylicacid (medium chain fatty acid), carnitine, acetyl-carnitine (C2) and butanoyl-carnitine (C4). The altered levels of isovaleryl-carnitine (C5) and propionyl-carnitine (C3) are indicative of altered uneven-chain fatty acid and/or altered amino acid catabolism. From the volcano plot (Fig. 1C), some of the most discriminatory (statistically significant) metabolites in the MELAS patients can be linked to stalled fatty acid catabolism/beta-oxidation. Many carnitine conjugates also increased in the MELAS group, however, the question arose as to how free carnitine can be increased as well in the MELAS group while it is used for detoxification and hence excreted in elevated levels as conjugates. For this to be possible, increased carnitine biosynthesis is needed. However, such a scenario would require one-carbon units in the form of sadenosylmethionine for the synthesis of carnitine via trimethyllysine. We thus speculate that due to the increased carnitine conjugates excreted in the urine of MELAS patients, one-carbon metabolism is affected in an effort to replenish the diminished carnitines. Thus, although many of the effected metabolites we report here can be linked to a redox imbalance and one-carbon metabolism, methylation and transsulfuration, our results indicate that stalled fatty acid oxidation was severely affected in this MELAS cohort.

Other metabolites detected as increased can be linked to different metabolic pathways. Increased amino acid catabolism (possibly through the activation of AMPK due to low energy levels) is supported by the increased levels of arginine, N2-succinyl-L-glutamic acid 5-semialdehyde, aspartic acid, and urea. Even though some of the patients received carnitine $(n=1)$ and arginine $(n=2)$ supplementation, our data was still supportive of stalled beta-oxidation (Fig. 4C) and increased 
amino acid catabolism (Fig. 4B), even when the patients receiving supplementation were excluded from the data analysis (data not shown). Altered levels of xanthine is supportive of affected purine pathway. Lastly, 2,3-butandiol would normally be associated with high level of alcohol consumption; however, this metabolite can also be expected in urine when high level of glucose (as detected in the MELAS patients) are present as it is further metabolized by the gut microbes (Elshaghabee et al., 2016; Venkataraman et al., 2014). Additionally the increased levels of 4-hydroxyvaleric acid could also be ascribed to gut microbial metabolism. Since seven of the nine MELAS patients demonstrated GI dysmotility, the altered levels of 2,3-butandiol and 4-hydroxyvaleric acid could indeed be due to altered gut metabolism on the measured urinary metabolome.

A study in 2015 (Hall et al., 2015) of renal involvement in mitochondrial disease included a cohort of 14 patients with the MELAS phenotype; NMR was utilized to identify six potential urinary markers ( $\mathrm{N}$-methylnicotinamide, hippurate, creatinine, 4-cresyl sulfate, glycolate, and a marker with unconfirmed identity, possibly 4-hydroxyphenylacetic acid). In our study, we detected and quantified $\mathrm{N}^{1}$-methylnicotinamide, hippurate, creatinine, glycolate, but none of these markers were significantly affected by MELAS ( $p>0.05$ and $d<0.8$ ) in our cohort. Lastly, our multi-platform approach resulted in the detection of 36 metabolites perturbed by MELAS compared to the six reported by Hall et al. (2015), whom only used a single analytical platform.

The strength of our study was the utilization of five different analytical platforms to generate the robust metabolomics data reported here. Of the five platforms employed, only three (GC-MS, NMR and LC-MS/MS) contributed to the majority of our metabolite list as illustrated in Figure 2. Although many discriminatory features were detected with untargeted LC-IM-MS, only four metabolites (in positive ionization mode) was identified with moderate confidence while none of the discriminatory features in negative ionization mode could be identified. Nevertheless, some of the 36 important metabolites were detected and identified as important by more than one platform (Fig. 2) due to 
their overlapping coverage of the metabolome, giving more credibility to the metabolite as being critical in the disease. One important metabolite were detected by both the NMR and LC-MS/MS (alanine) while the overlap between the GC-MS and NMR was two metabolites (lactic acid and pyruvic acid). In the light of these findings, we recommend using a multi-platform approach to analyze the metabolome in mitochondrial disease studies as the multiple platforms complement each other, rather than substitute for each other.

\section{CONCLUSION}

We have performed a multi-platform metabolomics investigation on two MELAS cohorts in order to better understand the metabolic alterations caused by the disease. To our knowledge, this is the first multi-platform metabolomics investigation of the MELAS phenotype. By combining the metabolic data sets generated with the multi-platform analytical approach, we were able to cover a larger part of the metabolome, which led to the identification of 36 metabolites (during the exploratory phase) that differed significantly between the patient and control group, compared to the six markers previously reported for the disease (Hall et al., 2015). During the validation phase, the 36 selected metabolites were able to separate a validation cohort of two MELAS patients completely from their respective control group.

Upon further investigation of our data, most of the disturbed metabolites could be explained by a redox imbalance caused by a defective OXPHOS system while stalled fatty acid oxidation prevailed as being particularly perturbed in the MELAS phenotype. Furthermore the intricate web of pathways consisting of the one-carbon metabolism, methylation cycle and trans-sulfuration pathway previously linked to PEO (Bao et al. 2016; Nikkanen et al., 2016) - can now, with our data, also be linked to the MELAS phenotype. Although redox imbalance has previously been associated with mitochondrial disease (Brière et al., 2004; Khan et al., 2014; Naviaux, 2014; Reinecke et al., 2009; 
Smeitink et al., 2006), our data confirms the global impact of mitochondrial disease on the metabolism, further contributing to its complexity.

The small number of MELAS patients used in this study (nine MELAS patients for the exploratory phase and only two patients for the validation phase) is a limitation of the study. Larger numbers of patients would have been preferential, although it was not available to this study. Also, all the MELAS patients used in this study had the m.3243A>G mutation - none had any of the numerous other MELAS-causing mutations. Thus the biosignature suggested here is specific for this single phenotype. Although further research is warranted, this study is the first to follow a comprehensive metabolomics analysis approach to identify metabolites altered by the MELAS phenotype. Understanding the metabolic alterations of MELAS could further our understanding of the disease and might lead to better treatment options.

\section{Acknowledgements}

The research was supported by a grant from the Technology Innovation Agency (TIA, Grant number Metabol. 01), South Africa. The research facilities were provided by the National Metabolomics Platform, North-West University, Potchefstroom Campus, Potchefstroom. 


\section{REFRENCES}

Bao, X.R., Ong, S.E., Goldberger, O., Peng, J., Sharma, R., Thompson, D.A., Vafai, S.B., Cox, A.G., Marutani, E., Ichinose, F., Goessling, W., Regev, A., Carr, S.A., Clish, C.B., Mootha, V.K., 2016. Mitochondrial dysfunction remodels one-carbon metabolism in human cells. elife 5, e10575.

Brière, J., Chrétien, D., Bénit, P., Rustin, P., 2004. Respiratory chain defects: what do we know for sure about their consequences in vivo? Biochim. Biophys. Acta Bioenergetics. 1659 (2), 172-177.

de Laat, P., Koene, S., van den Heuvel, L.P.W.J., Rodenburg, R.J.T., Janssen, M.C.H., Smeitink, J.A.M., 2012. Clinical features and heteroplasmy in blood, urine and saliva in 34 Dutch families carrying the m.3243A > G mutation. J Inherit Metab Dis. 35, 1059-1069.

Elshaghabee, F.M., Bockelmann, W., Meske, D., de Vrese, M., Walte, H.G., Schrezenmeir, J. \& Heller, K.J., 2016. Ethanol Production by Selected Intestinal Microorganisms and Lactic Acid Bacteria Growing under Different Nutritional Conditions. Frontiers in microbiology. 7, 47

Esterhuizen, K., van der Westhuizen, F.H. \& Louw, R., 2017. Metabolomics of mitochondrial disease . Mitochondrion. 35, 97-110.

Fiehn, O., Robertson, D., Griffin, J., van der Werf, M., Nikolau, B., Morrison, N., Sumner, L.W., Goodacre, R., Hardy, N.W., Taylor, C., Fostel, J., Kristal, B., Kaddurah-Daouk, R., Mendes, P., van Ommen, B., Lindon, J.C. \& Sansone, S., 2007. The metabolomics standards initiative (MSI). Metabolomics. 3, 175-178. 
Gorman, G.S., Schaefer, A.M., Ng, Y., Gomez, N., Blakely, E.L., Alston, C.L., Feeney, C., Horvath, R., Yu-Wai-Man, P., Chinnery, P.F., 2015. Prevalence of nuclear and mitochondrial DNA mutations related to adult mitochondrial disease. Ann. Neurol. 77 (5), 753-759.

Goto, Y., Nonaka, I. \& Horai, S., 1990. A mutation in the tRNA (Leu (UUR)) gene associated with the MELAS subgroup of mitochondrial encephalomyopathies. Nature. 348 (6302), 651-653

Hall, A.M., Vilasi, A., Garcia-Perez, I., Lapsley, M., Alston, C.L., Pitceathly, R.D., McFarland, R., Schaefer, A.M., Turnbull, D.M., Beaumont, N.J., 2015. The urinary proteome and metabonome differ from normal in adults with mitochondrial disease. Kidney Int. 87 (3), 610-622.

Kami, K., Fujita, Y., Igarashi, S., Koike, S., Sugawara, S., Ikeda, S., Sato, N., Ito, M., Tanaka, M., Tomita, M., 2012. Metabolomic profiling rationalized pyruvate efficacy in cybrid cells harboring MELAS mitochondrial DNA mutations. Mitochondrion. 12 (6), 644-653.

Khan, N.A., Auranen, M., Paetau, I., Pirinen, E., Euro, L., Forsström, S., Pasila, L., Velagapudi, V., Carroll, C.J., Auwerx, J., Suomalainen, A., 2014. Effective treatment of mitochondrial myopathy by nicotinamide riboside, a vitamin B3. EMBO Molecular Medicine. 6 (6), 721-731.

Manwaring, N., Jones, M.M., Wang, J.J., Rochtchina, E., Howard, C., Mitchell, P. \& Sue, C.M. , 2007. Population prevalence of the MELAS M.3243A>G mutation. Mitochondrion. 7 (3), 230-233.

Munnich, A., Rötig, A., Chretien, D., Saudubray, J., Cormier, V., Rustin, P., 1996. Clinical presentations and laboratory investigations in respiratory chain deficiency. Eur. J. Pediatr. 155, 262-274.

Naviaux, R.K., 2014. Metabolic features of the cell danger response. Mitochondrion 16, 7-17. 
Nikkanen, J., Forsström, S., Euro, L., Paetau, I., Kohnz, R.A., Wang, L., Chilov, D., Viinamäki, J., Roivainen, A., Marjamäki, P., 2016. Mitochondrial DNA replication defects disturb cellular dNTP pools and remodel one-carbon metabolism. Cell Metab. 23 (4), 635-648.

Pavlakis, S.G., Phillips, P.C., DiMauro, S., De Vivo, D.C. \& Rowland, L.P., 1984. Mitochondrial myopathy, encephalopathy, lactic acidosis, and strokelike episodes: a distinctive clinical syndrome . Annals of Neurology. 16 (4), 481-488.

Reinecke, C.J., Koekemoer, G., van der Westhuizen, F.H., Louw, R., Lindeque, J.Z., Mienie, L.J., Smuts, I., 2012. Metabolomics of urinary organic acids in respiratory chain deficiencies in children. Metabolomics 8(2), 264-283.

Ruiz-Pesini, E., Lott, M.T., Procaccio, V., Poole, J.C., Brandon, M.C., Mishmar, D., Yi, C., Kreuziger, J., Baldi, P. \& Wallace D.C., 2007. An enhanced MITOMAP with a global mtDNA mutational phylogeny. Nucleic Acids Research. 35, D823-D828.

Scaglia, F. \& Northrop, J.L., 2006. The mitochondrial myopathy encephalopathy, lactic acidosis with stroke-like episodes (MELAS) syndrome: a review of treatment options. CNS Drugs. 20 (6), 443-464.

Schaefer, A.M., McFarland, R., Blakely, E.L., He, L., Whittaker, R.G., Taylor, R.W., Chinnery, P.F. \& Turnbull, D.M., 2007. Prevalence of mitochondrial DNA disease in adults. Annals of Neurology. 63 (1), 35-39.

Smeitink, J.A., Zeviani, M., Turnbull, D.M., Jacobs, H.T., 2006. Mitochondrial medicine: a metabolic perspective on the pathology of oxidative phosphorylation disorders. Cell Metab. 3 (1), 9-13. 
Venkataraman, A., Rosenbaum, M.A., Werner, J.J., Winans, S.C. \& Angenent, L.T., 2014. Metabolite transfer with the fermentation product 2,3-butanediol enhances virulence by Pseudomonas aeruginosa. The ISME journal. 8 (6), 1210-1220. 


\section{A urinary biosignature for mitochondrial myopathy,}

encephalopathy, lactic acidosis and stroke like episodes (MELAS)

Table 1: Characteristics of the MELAS patients and healthy controls of the Nijmegen cohort used in this study (means \pm standard deviation).

\begin{tabular}{|l|c|c|}
\hline Characteristic & Patients & Controls \\
\hline Total, N & 9 & 29 \\
\hline Age, $\mathrm{y}$ & $31.4 \pm 11.6$ & $34.8 \pm 19.8$ \\
\hline Male & 6 & 10 \\
\hline Female & $3.2 \pm 5.2$ & $13.2 \pm 7.1$ \\
\hline Creatinine [mmol/L) & $570.2 \pm 279.4$ & $765.6 \pm 271.7$ \\
\hline Osmolality(mOsm/kg) & $44.9 \pm 18.7$ & $\mathrm{~N} / \mathrm{A}$ \\
\hline NMDAS Score & $81 \pm 0.19$ & $\mathrm{~N} / \mathrm{A}$ \\
\hline$\%$ m.3243A>G & & \\
\hline & & \\
\hline Symptoms (Number of patients presenting with symptoms) & $3(33.3 \%)$ & $\mathrm{N} / \mathrm{A}$ \\
\hline Cardiomyopathy & $7(77.8 \%)$ & $\mathrm{N} / \mathrm{A}$ \\
\hline Epilepsy & $9(100 \%)$ & $\mathrm{N} / \mathrm{A}$ \\
\hline Myopathy & $9(100 \%)$ & $\mathrm{N} / \mathrm{A}$ \\
\hline Strokes & $7(77.8 \%)$ & $\mathrm{N} / \mathrm{A}$ \\
\hline Gl dysmotility & $5(55.6 \%)$ & $\mathrm{N} / \mathrm{A}$ \\
\hline Diabetes & $0(0 \%)$ & $\mathrm{N} / \mathrm{A}$ \\
\hline Renal insufficiency & $9(100 \%)$ & $\mathrm{N} / \mathrm{A}$ \\
\hline Hearing loss & & \\
\hline & & $\mathrm{N}$ \\
\hline Medication (Number of patients exposed) & $1(11.1 \%)$ & $\mathrm{N} / \mathrm{A}$ \\
\hline Acetylsalicylic acid & $1(11.1 \%)$ & $\mathrm{N} / \mathrm{A}$ \\
\hline Angiotensin-converting-enzymeinhibitor (ACEinhibitor) & $2(22.2 \%)$ & $\mathrm{N} / \mathrm{A}$ \\
\hline Anticoagulant & $7(77.8 \%)$ & $\mathrm{N} / \mathrm{A}$ \\
\hline Antiepileptic & $1(11.1 \%)$ & $\mathrm{N} / \mathrm{A}$ \\
\hline Angiotensin Receptor Blockers (ARBs) & $2(22.2 \%)$ & $\mathrm{N} / \mathrm{A}$ \\
\hline Beta blockers & $1(11.1 \%)$ & $\mathrm{N} / \mathrm{A}$ \\
\hline Calcium antagonist & $1(11.1 \%)$ & $\mathrm{N} / \mathrm{A}$ \\
\hline Diuretic & $4(44.4 \%)$ & $\mathrm{N} / \mathrm{A}$ \\
\hline Insulin & $1(11.1 \%)$ & $\mathrm{N} / \mathrm{A}$ \\
\hline Laxative & $1(11.1 \%)$ & $\mathrm{N} / \mathrm{A}$ \\
\hline Statin & $1(11.1 \%)$ & $\mathrm{N} / \mathrm{A}$ \\
\hline Sulfanylurea derivative & & \\
\hline & & \\
\hline & & \\
\hline & & \\
\hline & & \\
\hline
\end{tabular}




\begin{tabular}{|l|r|l|} 
& & \\
\hline Supplements (Number of patients exposed) & & \\
\hline Arginine & $2(22.2 \%)$ & $\mathrm{N} / \mathrm{A}$ \\
\hline B12 carnitine & $1(11.1 \%)$ & $\mathrm{N} / \mathrm{A}$ \\
\hline Carnitine & $1(11.1 \%)$ & $\mathrm{N} / \mathrm{A}$ \\
\hline Coenzyme Q10 & $2(22.2 \%)$ & $\mathrm{N} / \mathrm{A}$ \\
\hline Folic acid & $1(11.1 \%)$ & $\mathrm{N} / \mathrm{A}$ \\
\hline Riboflavin & $2(22.2 \%)$ & $\mathrm{N} / \mathrm{A}$ \\
\hline
\end{tabular}

The m.3243A>G mutation load was determined in urinary epithelial cells as described earlier (de Laat et al., 2012). 
Table 2: Characteristics of the MELAS patients and healthy controls of the Helsinki cohort used in this study (means \pm standard deviation).

\begin{tabular}{|c|c|c|}
\hline Characteristic & Patients & Controls \\
\hline Total, N & 2 & 7 \\
\hline Age, $y$ & $54.7 \pm 2.2$ & $38.5 \pm 13.6$ \\
\hline Creatinine [mmol/L) & $7.4 \pm 2.4$ & $16.2 \pm 2.9$ \\
\hline Osmolality(mOsm/kg) & $504.5 \pm 187.4$ & $755.0 \pm 69.2$ \\
\hline$\%$ m.3243A $>$ G urine & $71.5 \pm 20.5$ & $\mathrm{~N} / \mathrm{A}$ \\
\hline$\%$ m.3243A>G blood & $21.5 \pm 9.2$ & $\mathrm{~N} / \mathrm{A}$ \\
\hline \multicolumn{3}{|l|}{ Symptoms (Number of patients presenting with symptoms) } \\
\hline Ataxia & $1(50 \%)$ & $\mathrm{N} / \mathrm{A}$ \\
\hline Cardiomyopathy & $2(100 \%)$ & $\mathrm{N} / \mathrm{A}$ \\
\hline Depression & $2(100 \%)$ & $\mathrm{N} / \mathrm{A}$ \\
\hline Diabetes & $2(100 \%)$ & $\mathrm{N} / \mathrm{A}$ \\
\hline Hearing loss & $2(100 \%)$ & $\mathrm{N} / \mathrm{A}$ \\
\hline Hyperaldosteronism & $1(50 \%)$ & $\mathrm{N} / \mathrm{A}$ \\
\hline Myopathy & $1(50 \%)$ & N/A \\
\hline Migraine-type headache & $1(50 \%)$ & N/A \\
\hline+8 & $1(50 \%)$ & $\mathrm{N} / \mathrm{A}$ \\
\hline \multicolumn{3}{|l|}{ Medication (Number of patients exposed) } \\
\hline Acetylsalicylic acid & $1(50 \%)$ & N/A \\
\hline Angiotensin-converting-enzyme inhibitor (ACE inhibitor) & $1(50 \%)$ & N/A \\
\hline Beta blocker & $1(50 \%)$ & N/A \\
\hline Benzodiazepines & $2(100 \%)$ & N/A \\
\hline Insulin & $2(100 \%)$ & $\mathrm{N} / \mathrm{A}$ \\
\hline Metformin & $1(50 \%)$ & N/A \\
\hline Sulfanylurea derivative & $1(50 \%)$ & N/A \\
\hline 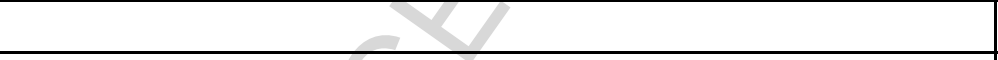 & & \\
\hline \multicolumn{3}{|l|}{ Supplements (Number of patients exposed) } \\
\hline Calcium & $1(50 \%)$ & N/A \\
\hline Coenzyme Q10 & $1(50 \%)$ & $\mathrm{N} / \mathrm{A}$ \\
\hline Iron & $1(50 \%)$ & $\mathrm{N} / \mathrm{A}$ \\
\hline Magnesium & $2(100 \%)$ & $\mathrm{N} / \mathrm{A}$ \\
\hline Omega & $2(100 \%)$ & $\mathrm{N} / \mathrm{A}$ \\
\hline Vitamin B & $1(50 \%)$ & $\mathrm{N} / \mathrm{A}$ \\
\hline Vitamin D & $1(50 \%)$ & $\mathrm{N} / \mathrm{A}$ \\
\hline
\end{tabular}

The m.3243A>G mutation load was determined in urinary epithelial cells. 
Table 3. List of $\mathbf{3 6}$ metabolites that differed significantly between MELAS patients and controls in the Nijmegen cohort

\begin{tabular}{|c|c|c|c|c|c|}
\hline & Direction & $\begin{array}{c}\text { P-Value } \\
\text { (Student } \\
\text { t-test) }\end{array}$ & $\begin{array}{c}\text { D-Value } \\
\text { (Effect size) }\end{array}$ & $\begin{array}{c}\text { Level of } \\
\text { identification }\end{array}$ & Platform \\
\hline \multicolumn{6}{|l|}{ AMINO ACIDS } \\
\hline Alanine & $\uparrow$ & 0.001 & 1.258 & 1 & NMR (LC-MS/MS) \\
\hline Arginine & $\uparrow$ & 0.015 & 0.962 & 1 & LC-MS/MS \\
\hline Aspartic acid & $\uparrow$ & 0.019 & 0.872 & 1 & LC-MS/MS \\
\hline Cysteine & $\uparrow$ & 0.001 & 1.244 & 1 & LC-MS/MS \\
\hline Isoleucine & $\uparrow$ & 0.001 & 1.050 & 1 & NMR \\
\hline Leucine & $\uparrow$ & 0.004 & 1.007 & 1 & NMR \\
\hline Lysine & $\uparrow$ & 0.014 & 0.895 & 1 & NMR \\
\hline $\begin{array}{l}\text { N2-Succinyl-L-glutamic acid } \\
\text { 5-semial dehyde }\end{array}$ & $\uparrow$ & 0.004 & 1.052 & 3 & LC-IM-MS (+) \\
\hline $\mathrm{N}$-Acetyltryptophan & $\uparrow$ & 0.003 & 1.118 & 3 & LC-IM-MS (+) \\
\hline Proline & $\uparrow$ & 0.004 & 1.123 & 1 & NMR \\
\hline Sarcosine & $\downarrow$ & 0.031 & 0.853 & 1 & GC-MS \\
\hline Taurine & $\uparrow$ & 0.014 & 0.924 & 3 & GC-MS \\
\hline Trimethylglycine (Betaine) & $\uparrow$ & 0.003 & 1.100 & 1 & NMR \\
\hline \multicolumn{6}{|l|}{ ORGANICACIDS } \\
\hline 3-Hydroxybutyric acid & $\uparrow$ & 0.011 & 0.993 & 1 & GC-MS \\
\hline 4-Hydroxyglutaric acid & $\uparrow$ & 0.007 & 1.033 & 3 & GC-MS \\
\hline Acetoaceticacid & $\uparrow$ & 0.037 & 0.810 & 1 & NMR \\
\hline Butyric/valeric acid & $\uparrow$ & 0.000 & 1.107 & 2 & NMR \\
\hline Caproic/caprylic acid & $\uparrow$ & 0.000 & 1.225 & 2 & NMR \\
\hline Citricacid & $\uparrow$ & 0.004 & 1.134 & 1 & NMR \\
\hline Glycerol & $\downarrow$ & 0.002 & 1.183 & 3 & GC-MS \\
\hline Lactic acid & $\uparrow$ & 0.004 & 1.085 & 1 & GC-MS (NMR) \\
\hline Pyruvic acid & $\uparrow$ & 0.014 & 0.987 & 1 & NMR (GC-MS) \\
\hline$r a$ & & & & & \\
\hline \multicolumn{6}{|l|}{ CARNITINES } \\
\hline Carnitine & $\uparrow$ & 0.000 & 1.068 & 1 & NMR \\
\hline C2-Carnitine & $\uparrow$ & 0.000 & 1.083 & 1 & LC-MS/MS \\
\hline C3-Carnitine & $\uparrow$ & 0.000 & 1.017 & 1 & LC-MS/MS \\
\hline C4-Carnitine & $\uparrow$ & 0.002 & 0.923 & 1 & LC-MS/MS \\
\hline C5-Carnitine & $\uparrow$ & 0.000 & 0.895 & 1 & LC-MS/MS \\
\hline \multicolumn{6}{|l|}{ OTHER } \\
\hline 6-Azathymine & $\downarrow$ & 0.006 & 0.973 & 3 & GC-MS \\
\hline 2,3-Butanediol glucoside & $\uparrow$ & 0.002 & 1.139 & 3 & LC-IM-MS (+) \\
\hline Butanal & $\uparrow$ & 0.015 & 0.834 & 3 & GC-MS \\
\hline Glucose & $\uparrow$ & 0.000 & 1.225 & 1 & NMR \\
\hline
\end{tabular}




\begin{tabular}{|l|c|c|c|c|c|}
\hline Hydroxylamine & $\downarrow$ & 0.002 & 1.081 & 3 & GC-MS \\
\hline Resolvin D2 & $\uparrow$ & 0.015 & 0.877 & 3 & LC-IM-MS (+) \\
\hline TMAO & $\uparrow$ & 0.004 & 0.844 & 1 & NMR \\
\hline Urea & $\uparrow$ & 0.024 & 0.878 & 1 & NMR \\
\hline Xanthine & $\uparrow$ & 0.011 & 0.856 & 1 & NMR \\
\hline
\end{tabular}

If a metabolite was detected as significant by more than one analytical platform, the second platform is indicated in the table in brackets. $\uparrow$ means the metabolite is increased in the MELAS patients, relative to the Controls. $\downarrow$ means the metabolite is decreased in the MELAS patients, relative to the Controls. 
A
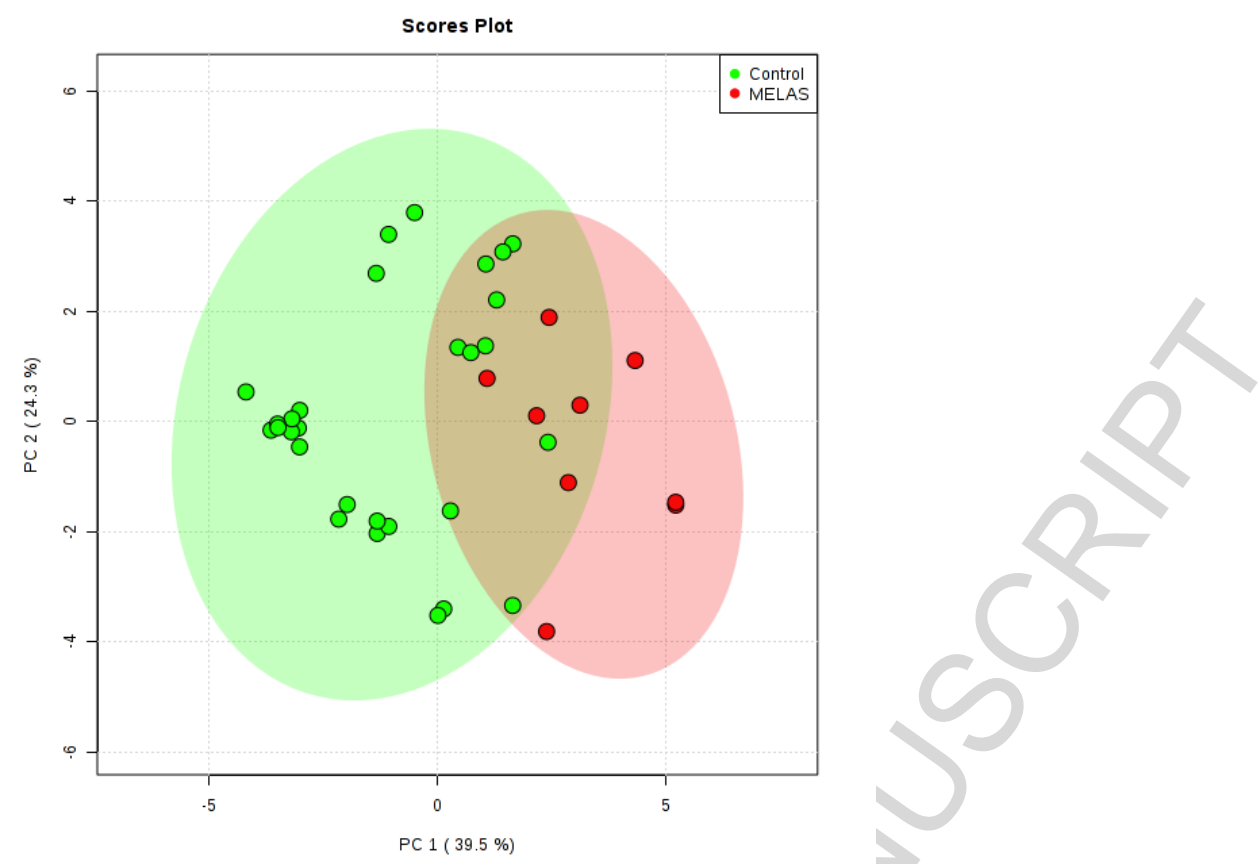

B

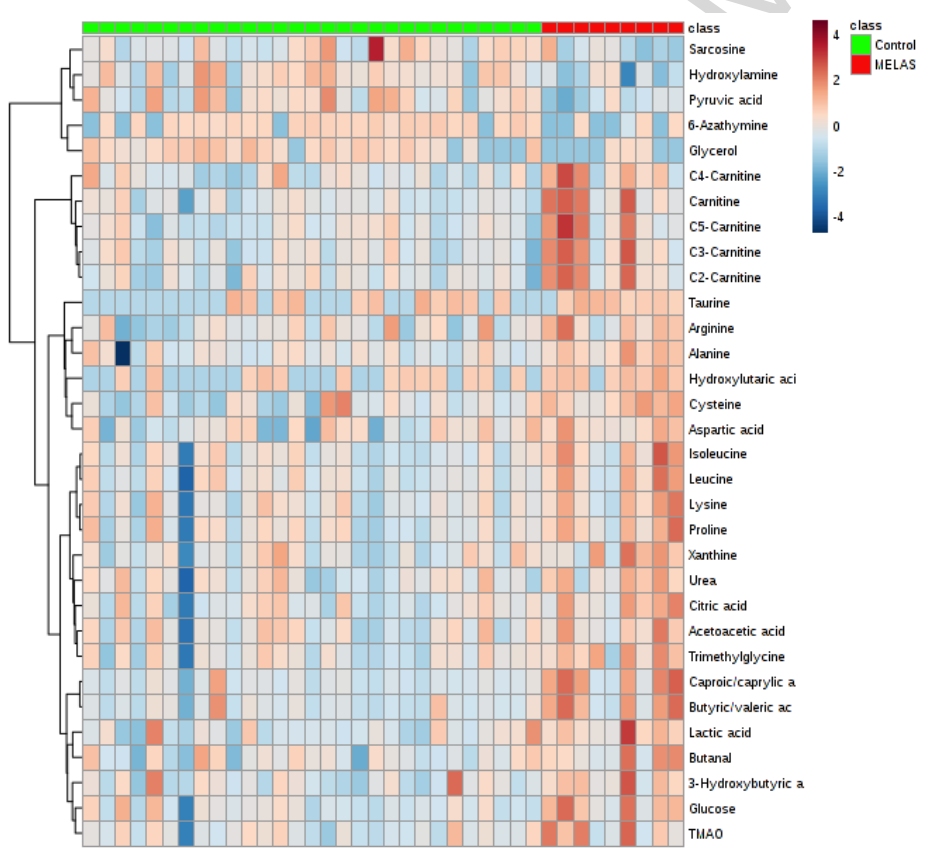


C

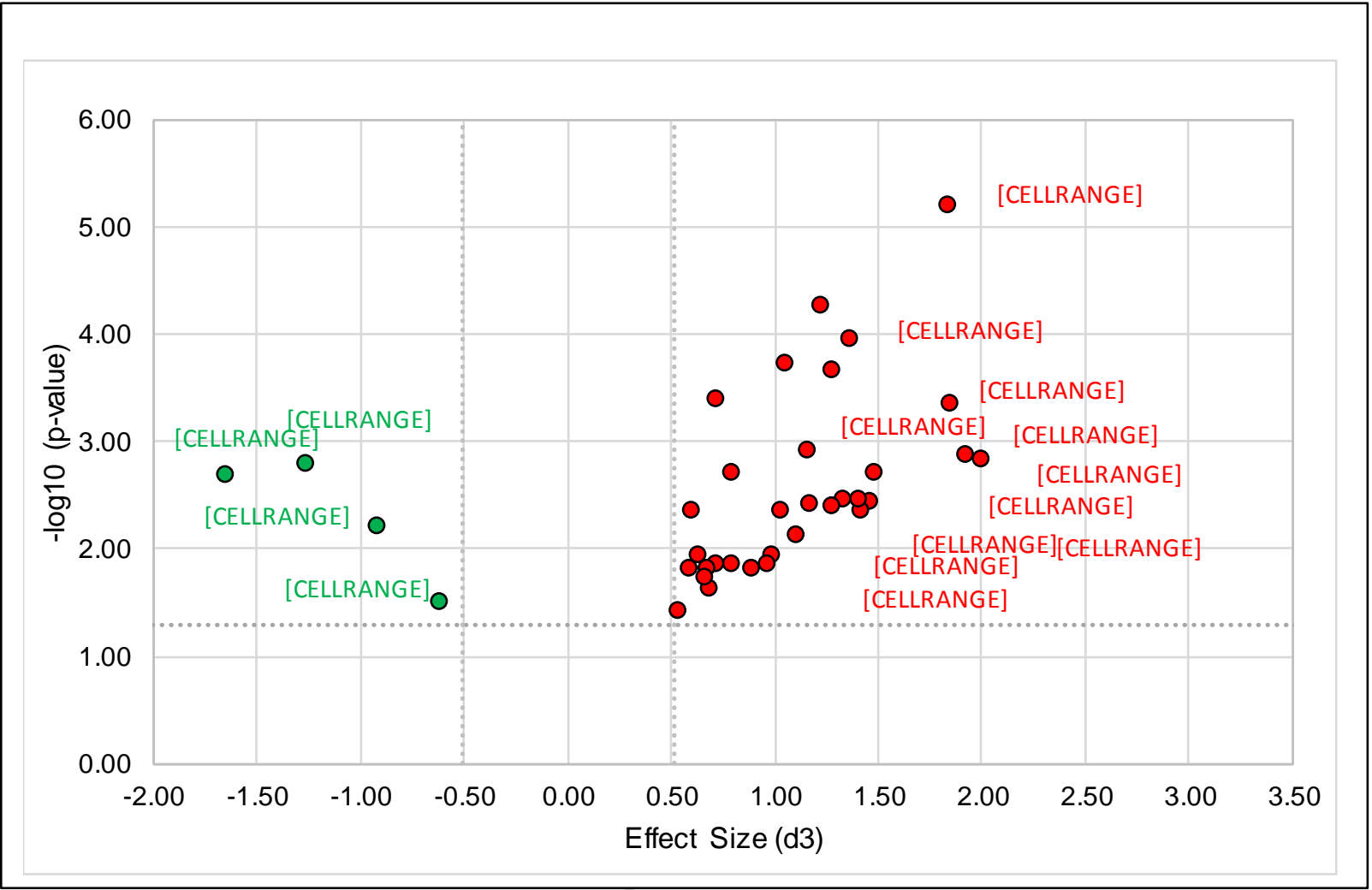

Fig 1. Differences between Controls and MELAS patients of the exploration (Nijmegen) cohort using the 36 selected variables. A) principal component analysis score (PCA) plot. Sample grouping are shown in the PCA with $90 \%$ confidence ellipses; B) heatmap; C) volcano plot. 


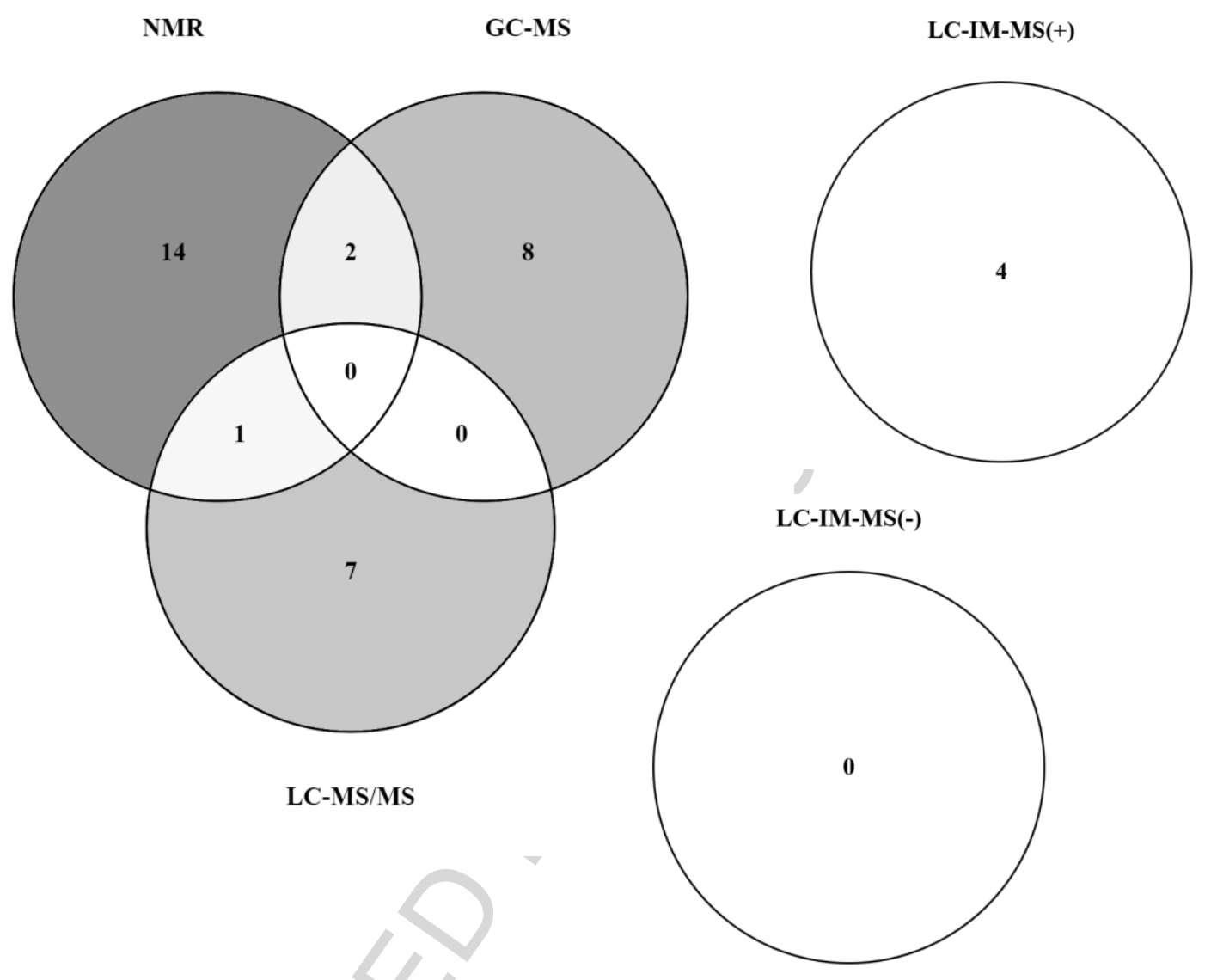

Fig 2. Venn diagram illustrating the contribution of each analytical platform to the detection of the 36 important metabolites. Five analytical platforms were used to analyze the metabolome of the MELAS patients and controls. Although a large number of features were detected using each platform, only the metabolites that differed significantly between the MELAS and controls groups and that could be identified/partially identified were considered as "important", thus forming part of the final metabolic fingerprint consisting of 36 metabolites. 
A
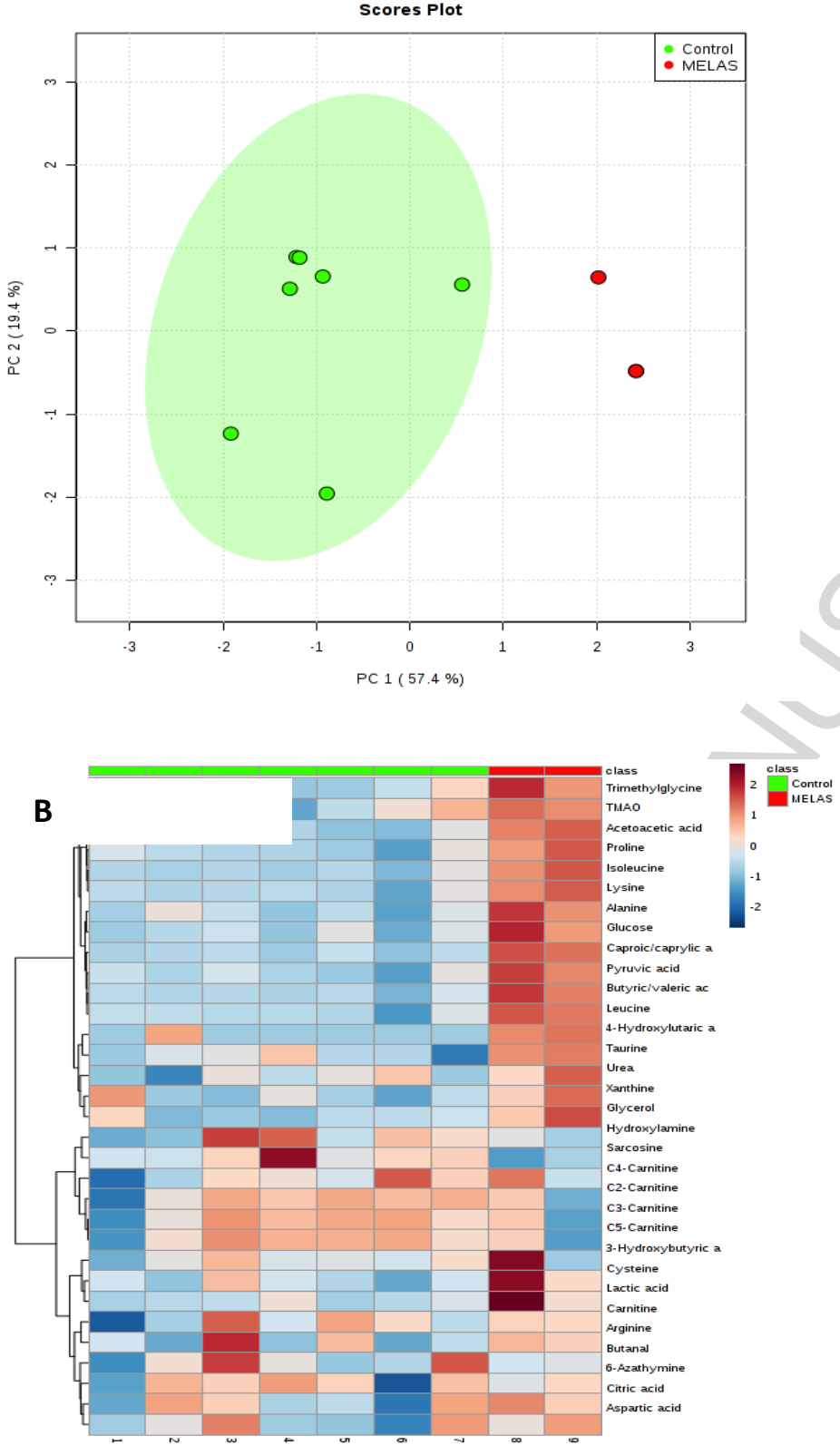

Fig 3. Differences between Controls and MELAS patients of the validation (Helsinki) cohort using the 36 selected variables. A) principal component analysis score (PCA) - since this cohort only contains two MELAS samples, an ellipse could not be drawn for the patient group, although the two samples clearly grouped well away from the controls, in dicating natural separation. Sample grouping are shown in the PCA with $90 \%$ confidence ellipses; B) heatmap 


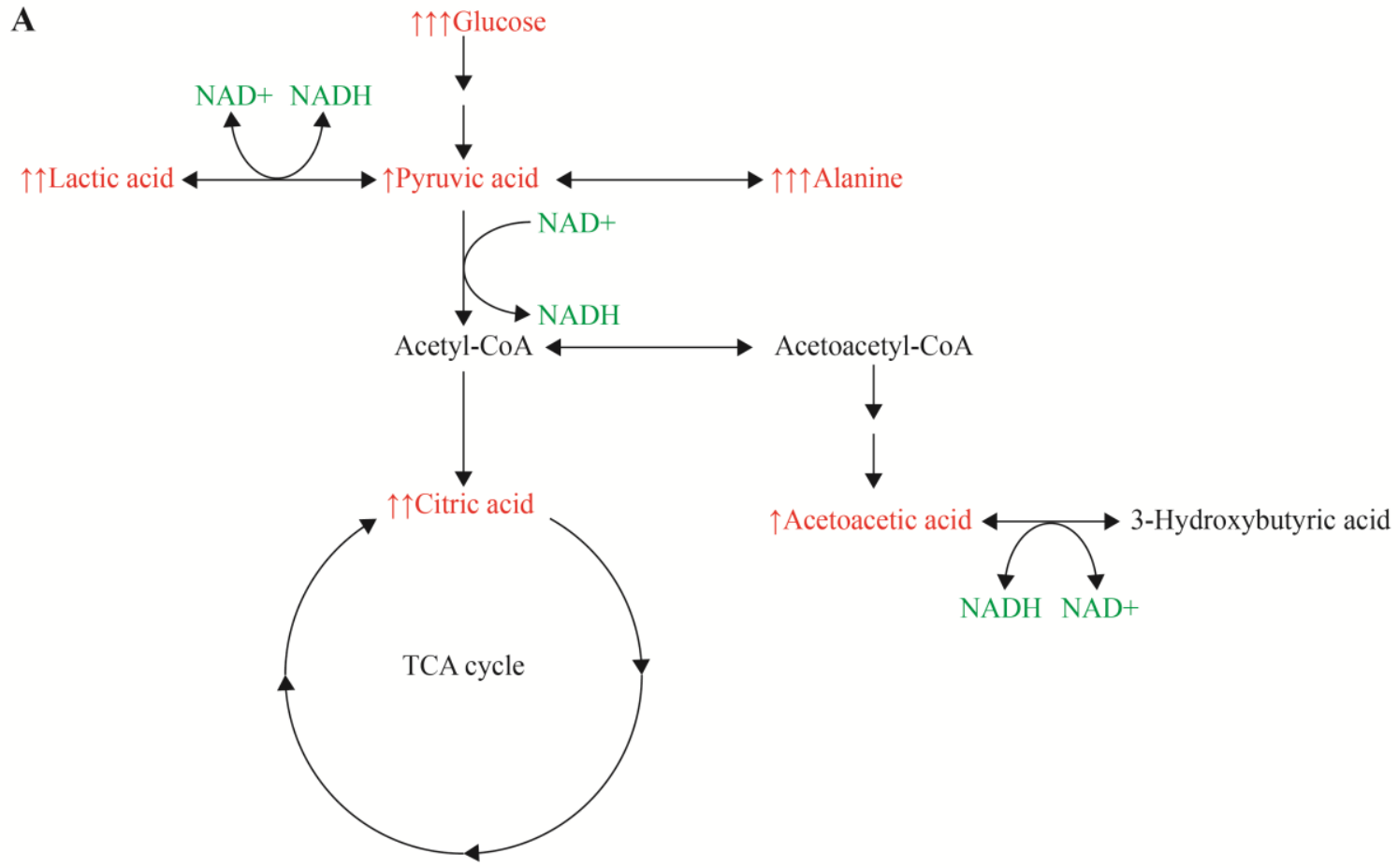

B

\section{Proteins}

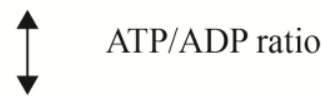

$\uparrow$ Amino acids

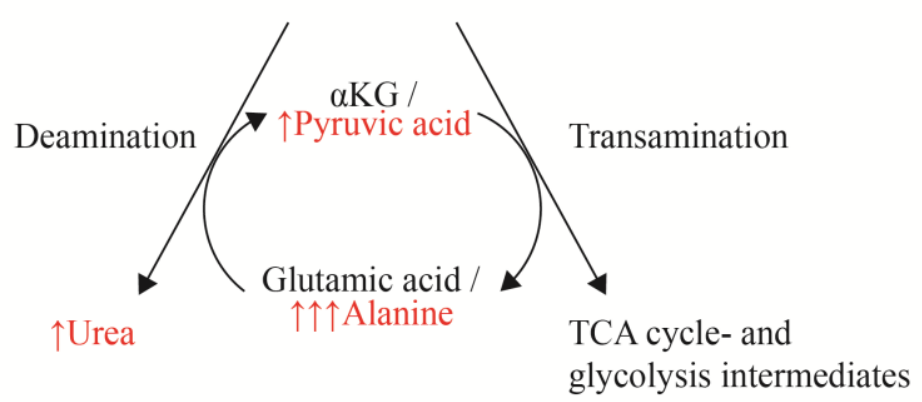


C

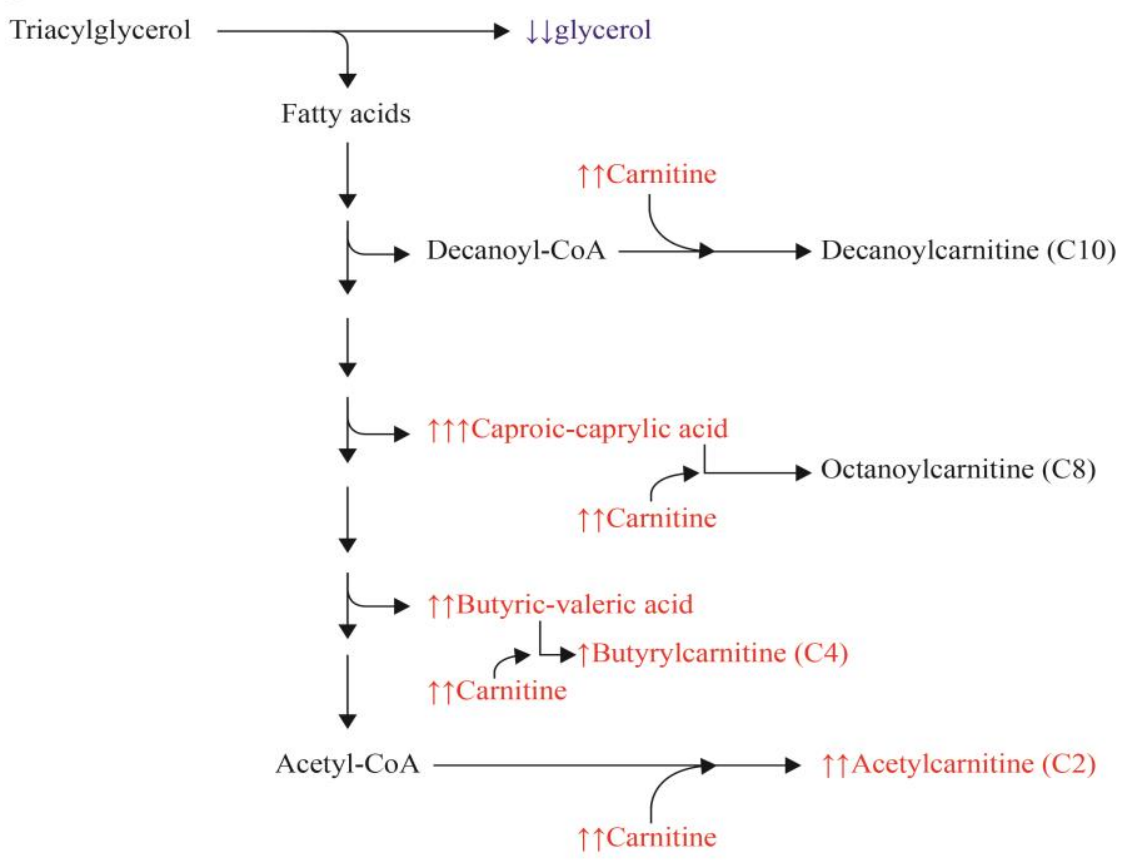

D

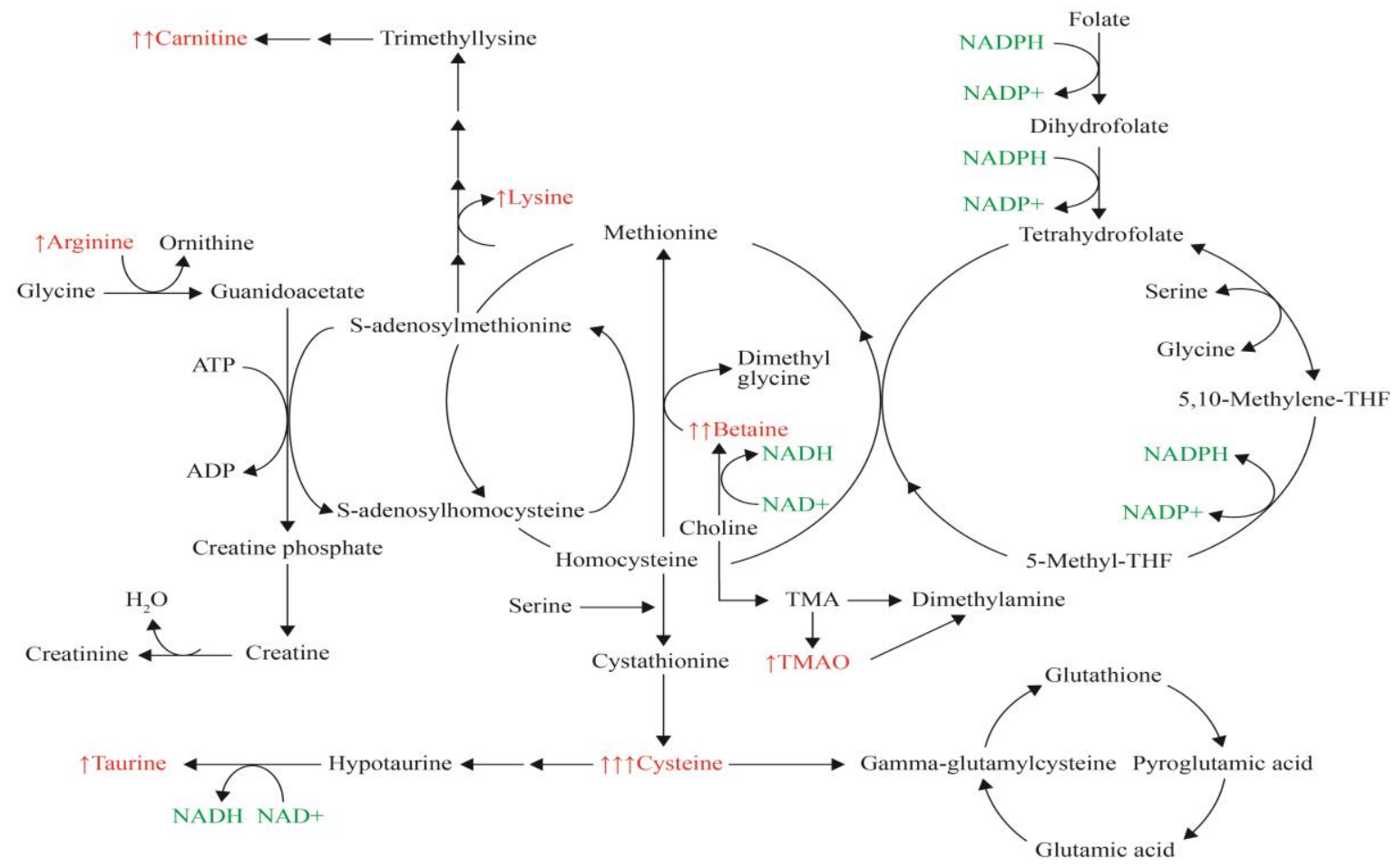

Fig 4. Schematic representation of the altered metabolism detected in the MELAS patients compared to healthy controls. A) altered glucose metabolism, B) protein catabolism, C) lipid catabolism, D) one-carbon metabolism, methylation cycle and the transsulfuration route. Metabolites significantly increased by MELAS (compared to the controls) are indicated in red, those significantly decreased are indicated in blue. $\uparrow=$ metabolite is increased $(d<1.0) ; \uparrow \uparrow=$ moderate 
increase $(d<1.2)$; $\uparrow \uparrow \uparrow=$ highly increase $(d>1.2)$ and $\downarrow \downarrow=$ moderate decrease $(d<1.2)$. Cofactor dependent reactions are indicated in green. 


\section{Highlights}

- We used a comprehensive metabolomics approach to study the altered urinary metabolome of two MELAS cohorts

- The first cohort were used in an exploratory phase, identifying 36 metabolites that were significantly perturbed by the disease

- The 36 selected metabolites were able to separate a validation cohort of MELAS patients completely from their respective control group

- Many of the perturbed metabolites could be linked to an altered redox state, fatty acid catabolism and one-carbon metabolism

- We show that urine may be a useful source for disease-specificmetabolomics data 